\title{
Is Cheyne-Stokes respiration friend or foe of heart failure?
}

I agree with Dr MT Naughton's interesting opinion expressed in 'Cheyne-Stokes respiration: friend or foe?' in his recent opinion in Thorax. ${ }^{1}$ Central sleep apnoea with Cheyne-Stokes respiration (CSA-CSR) indeed has multiple features more likely to be compensatory than injurious in heart failure (HF). His view, however, seems to lack insight into the heart-lung interaction in CSA-CSR, and reminds me of the fundamental question of 'why does the heartbeat synchronise with respiratory rhythm?'.

In polygraphic recording in a $\mathrm{HF}$ patient with CSA-CSR (figure 1A), heartbeats are clustered during the hyperventilation period and scattered during the apnoeic period of CSR-CSA with cyclelength of approximately $90 \mathrm{~s}$. This phenomenon is known as an entrainment of heartbeats, and the fluctuation in ventilation during CSA-CSR amplifies the oscillation in heart rate even in the absence of hypoxia or arousal from sleep. ${ }^{3}$ The stroke volume has been reported to increase $25 \%$ during the hyperventilation period compared with the apnoeic period, ${ }^{4}$ with the cardiac output to be thereby increased by at least $25 \%$.

Despite the different time-constant, the entrainment of heartbeats in CSA-CSR could be superimposed on respiratory sinus arrhythmia (RSA) featuring heartbeats clustered during inspiration, and sparse during expiration (figure 1B). We have already demonstrated in an experimental animal study, however, that efficacy of pulmonary gas exchange is improved by RSA. ${ }^{5}$

I consider that RSA, as well as CSA-CSR in HF, might not simply be the secondary products of other known reflexes, but could well play physiological roles. Supposing the teleology of the coexistence of CSA-CSR with HF is to improve the efficacy of pulmonary gas exchange, it might function to curb energy expenditure by reducing unnecessary heartbeats during the apnoeic period and unnecessary ventilation during the waning phase of heartbeats for failing heart with easily fatigued respiratory muscles. 
A

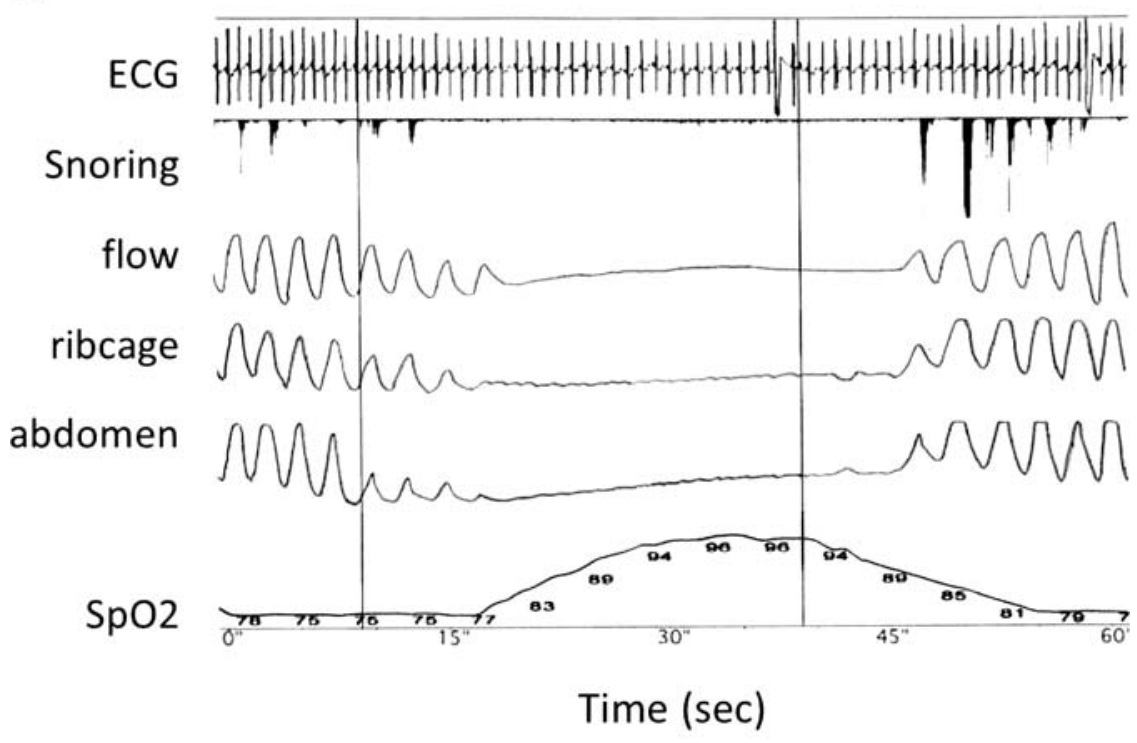

B

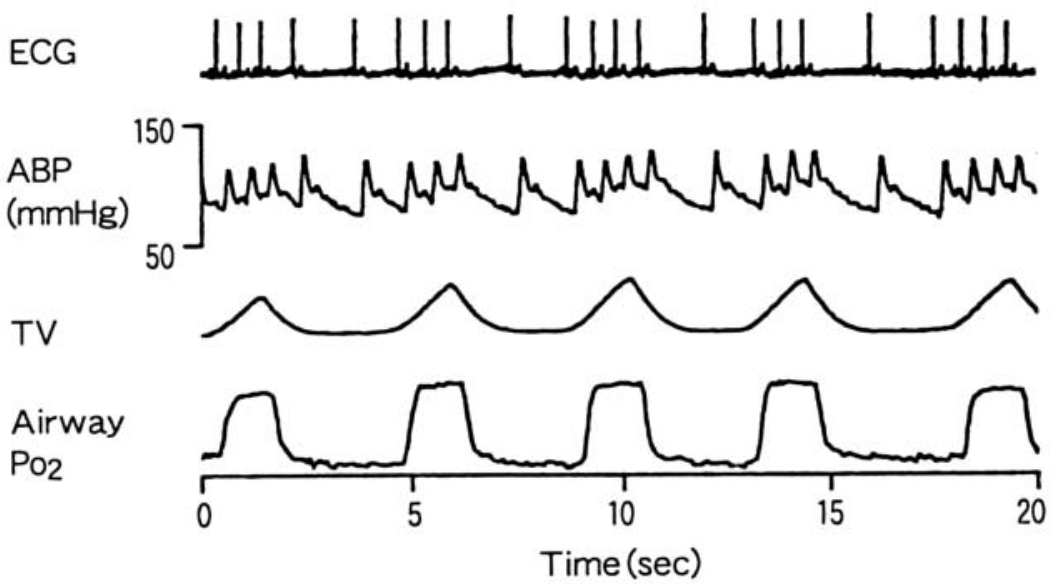

Figure 1 Polygraphic recordings showing Central sleep apnoea with Cheyne-Stokes respiration in a heart failure (HF) patient (A) and respiratory sinus arrhythmia in a conscious dog (B). ECG, snoring, oronasal flow, ribcage motion, abdominal motion and arterial oxygen saturation (Sp02) are displayed in (A), and ECG, arterial blood pressure, tidal volume and airway 02 tension (PO2) are displayed in (B). Clustering of heartbeats during the hyperventilation period, and scattering during the apnoeic period, are seen in (A) and, despite the different time-constant, clustering during inspiration and scattering during expiration are seen in (B). (A) is recorded in a HF patient during sleep, and (B) is recorded in a conscious dog lying quietly, and breathing via an endotracheal tube inserted through a permanent tracheostomy.

The majority of HF patients are complicated by CSA-CSR, which is more enhanced during sleep than wakefulness, and during exercise than resting. ${ }^{6}$ Cardiopulmonary efficacy must be improved to compensate for the inability to adopt the physiological changes induced by sleep and exercise in HF. And this is what has reminded me of the wisdom of nature in the RSA heart-lung interaction.

If CSA-CSR in HF could play an active physiological role, I agree with $\mathrm{Dr}$ Naughton that CSA-CSR is friend (compensatory), not foe (injurious and, thus, a possible therapeutic target), of HF and that the mechanisms of this phenomenon must be further investigated.

\section{Fumihiko Yasuma}

Correspondence to Dr Fumihiko Yasuma, Department of Cardilogy, Suzuka National Hospital, 3-2-1 Kasado, Suzuka, Mie prefecture 513-8501, Japan; f-yasuma@mtb.biglobe.ne.jp

\section{Competing interests None.}

Patient consent Obtained.

Ethics approval Standard and routine

polysomnography in the heart failure patient was used in this paper.

Provenance and peer review Not commissioned; internally peer reviewed.

To cite Field JK, Baldwin D, Brain K, et al. Thorax 2013, 68, 106-108.

Accepted 27 September 2012

Published Online First 23 October 2012

Thorax 2013;68:106-108.

doi:10.1136/thoraxjnl-2012-202725

\section{REFERENCES}

1 Naughton MT. Cheyne-Stokes respiration: friend or foe? Thorax 2012;67:357-60.

2 Yasuma F, Hayano J. Respiratory sinus arrhythmiawhy does the heartbeat synchronize with respiratory rhythm? Chest 2004;125:683-90.

3 Lorenzi-Filho G, Dajani HR, Leung RST, et al. Entrainment of blood pressure and heart rate oscillations by periodic breathing. Am J Respir Crit Care Med 1999:159:1147-54.

4 Maze SS, Kotler MN, Parry WR. Doppler evaluation of changing cardiac dynamics during Cheyne-Stokes Respiration. BMJ 1989;95:525-9.

5 Hayano J, Yasuma F, Okada A, et al. Respiratory sinus arrhythmia: a phenomenon improving pulmonary gas exchange and circulatory efficiency. Circulation 1996;94:842-7.

6 Olson LJ, Arruda-Olson AM, Somers VK, et al. Exercise oscillatory ventilation -instability of breathing control associated with advanced heart failure. Chest 2008;133:474-80. 